\title{
Part of Speech Tagger for Assamese Text
}

\author{
Navanath Saharia \\ Department of CSE \\ Tezpur University \\ India - 784028 \\ Dhrubajyoti Das \\ Department of CSE \\ Tezpur University \\ India - 784028 \\ Utpal Sharma \\ Department of CSE \\ Tezpur University \\ India - 784028
}

\author{
Jugal Kalita \\ Department of CS \\ University of Colorado \\ Colorado Springs - 80918 \\ kalita@eas.uccs.edu
}

\begin{abstract}
Assamese

is

a morphologically rich, agglutinative and relatively free word order Indic language. Although spoken by nearly 30 million people, very little computational linguistic work has been done for this language. In this paper, we present our work on part of speech (POS) tagging for Assamese using the well-known Hidden Markov Model. Since no well-defined suitable tagset was available, we develop a tagset of 172 tags in consultation with experts in linguistics. For successful tagging, we examine relevant linguistic issues in Assamese. For unknown words, we perform simple morphological analysis to determine probable tags. Using a manually tagged corpus of about 10000 words for training, we obtain a tagging accuracy of nearly $87 \%$ for test inputs.
\end{abstract}

\section{Introduction}

Part of Speech (POS) tagging is the process of marking up words and punctuation characters in a text with appropriate POS labels. The problems faced in POS tagging are many. Many words that occur in natural language texts are not listed in any catalog or lexicon. A large percentage of words also show ambiguity regarding lexical category.

The challenges of our work on POS tagging for Assamese, an Indo-European language, are compounded by the fact that very little prior computational linguistic exists for the language, though it is a national language of India and spoken by over 30 million people. Assamese is a morphologically rich, free word order, inflectional language. Although POS tagged annotated corpus for some of the Indian languages such as Hindi, Bengali, and Telegu (SPSAL, 2007) have become available lately, a POS tagged corpus for Assamese was unavailable till we started creating one for the work presented in this paper. Another problem was that a clearly defined POS tagset for Assamese was unavailable to us. As a part of the work reported in this paper, we have developed a tagset consisting of 172 tags, using this tagset we have manually tagged a corpus of about ten thousand Assamese words.

In the next section we provide a brief relevant linguistic background of Assamese. Section 3 contains an overview of work on POS tagging. Section 4 describes our experimental setup. In Section 5, we analyse the result of our work and compare the performance with other models. Section 6 concludes this paper.

\section{Linguistic Characteristics of Assamese}

In Assamese, secondary forms of words are formed through three processes: affixation, derivation and compounding. Affixes play a very important role in word formation. Affixes are used in the formation of relational nouns and pronouns, and in the inflection of verbs with respect to number, person, tense, aspect and mood. For example, Table 1 shows how a relational noun দেভতা (deutA: father) is inflected depending on number and person (Goswami, 2003). Though Assamese is relatively free word order, yet the predominant word order is subject-object-verb (SOV).

The following paragraphs describe just a few of the many characteristics of Assamese text that make the tagging task complex.

- Depending on the context, even a common word may have different POS tags. For example: If কাবণে (kArane), দবে (dare), নিমিত্তে (nimitte), হেতু (hetu), etc., are placed after pronominal adjective, they are considered conjunction and if placed after 
Table 1: Personal definitives are inflected on person and number

\begin{tabular}{|c|c|c|}
\hline Person & Singular & Plural \\
\hline $\begin{array}{l}1^{\text {st }} \\
\text { প্রথম }\end{array}$ & $\begin{array}{l}\text { My father } \\
\text { দোব দেউতা } \\
\text { mor deutA }\end{array}$ & $\begin{array}{l}\text { Our father } \\
\text { আমাব দেউতা } \\
a A m A r \text { deutA }\end{array}$ \\
\hline $\begin{array}{l}2^{\text {nd }} \\
\text { মান্য মধ্যম }\end{array}$ & $\begin{array}{l}\text { Your father } \\
\text { তোমাব দেউতাবা } \\
\text { tomAr deutArA }\end{array}$ & $\begin{array}{l}\text { Your father } \\
\text { তোমালোকব দেউতাবা } \\
\text { tomAlokar deutArA }\end{array}$ \\
\hline $\begin{array}{l}2^{\text {nd }}, \text { Familiar } \\
\text { তুচ্ছ মধ্যম }\end{array}$ & $\begin{array}{l}\text { Your father } \\
\text { তোব দেউতাব } \\
\text { tor deutAr }\end{array}$ & $\begin{array}{l}\text { Your father } \\
\text { তহতঁব দেউতাব } \\
\text { tahator deutAr }\end{array}$ \\
\hline $\begin{array}{l}3^{\text {rd }} \\
\text { তৃতীয় }\end{array}$ & $\begin{array}{l}\text { Her father } \\
\text { তাইব দেভতাক } \\
\text { tAir deutAk }\end{array}$ & $\begin{array}{l}\text { Their father } \\
\text { সিহঁতব দে৬তাক } \\
\text { sihator deutAk }\end{array}$ \\
\hline
\end{tabular}

noun or personal pronoun they are considered particle. For example,

এই কাবণে মই নগলোঁ।

$\mathrm{TF}^{1}$ : ei kArane moi nagalo.

This + why + I+ did not go.

$\mathrm{ET}^{2}$ : This is why I did not go.

বামব কাবণে মই নগলোঁ।

TF : rAmar kArane moi nagalo.

Ram's + because of $+I+$ did not go

ET : I did not go because of Ram.

In the first sentence কাবণে (kArne) is placed after pronominal adjective এই (ei); so kArne is considered conjunction. But in the second sentence kArne is placed after noun বাম $(R A m)$, and hence kArne is considered particle.

- Some prepositions or particles are used as suffix if they occur after noun, personal pronoun or verb. For example,

সিহে গৈগিল। TF: sihe goisil.

ET : Only he went.

Actually হে (he : only) is a particle, but it is merged with the personal pronoun সি $(s i)$.

- An affix denoting number, gender or person, can be added to an adjective or other category word to create a noun word. For example,

ধুনীয়াজনী ইৈ আহিছা।

TF : dhuniyAjoni hoi aAhisA.

ET : You are looking beautiful.

Here ধুনীয়া (dhuniyA : beautiful) is an adjective, but after adding feminine suffix जनी the whole constituent becomes a noun word.

\footnotetext{
${ }^{1} \mathrm{TF}:$ Transliterated Assamese Form

${ }^{2}$ ET : Aproximate English Translation
}

- Even conjunctions can be used as other part of speech.

হবি আবু যদু ভায়েক ককায়েক।

TF : Hari aAru Jadu bhAyek kokAyek.

ET : Hari and Jadu are brothers.

যোরাকালি বাতিব ঘটনাটোরে বিষয়টোক আবু অধিক বহস্যজনক কবি তুলিলে।

TF : JowAkAli rAtir ghotonAtowe bishoitok aAru adhik rahashyajanak kori tulile.

ET : The last night incident has made the matter more mysterious.

The word আবু (aAru : and) shows ambiguity in these two sentences. In the first, it is used as conjunction (i.e. Hari and Jadu) and in the second, it is used as adjective of adjective.

\section{Related Work}

Several approaches have been used for building POS taggers. Two main approaches are supervised and unsupervised. Both supervised and unsupervised tagging can be of three sub-types. They are rule based, stochastic based and neural network based. There are number of pros and cons for each of these methods. The most common stochastic tagging technique is Hidden Markov Model (HMM).

During the last two decades, many different types of taggers have been developed, especially for corpus rich languages such as English. Nevertheless, due to relatively free word order, agglutinative nature, lack of resources and the general lateness in entering the computational linguistics field in India, reported tagger development work on Indian languages is relatively scanty. Among reported works, Dandapat (2007) developed a hybrid model of POS tagging by combining both supervised and unsupervised stochastic techniques. Avinesh and Karthik (2007) used conditional random field and transformation based learning. The heart of the system developed by Singh et al. (2006) for Hindi was the detailed linguistic analysis of morphosyntactic phenomena, adroit handling of suffixes, accurate verb group identification and learning of disambiguation rules. Saha et al. (2004) developed a system for machine assisted POS tagging of Bangla corpora. Pammi and Prahllad (2007) developed a POS tagger and chunker using Decision Forests. This work explored different methods for POS tagging of Indian languages using sub-words as units. Generally, most POS taggers for Indian langauages use 
morphological analyzer as a module. However, building morphological analyzer of a particular Indian language is a very difficult task.

\section{Our Approach}

We have used a Assamese text corpus (Corpus Asm) of nearly 300,000 words from the online version of the Assamese daily Asomiya Pratidin (Sharma et al., 2008). The downloaded articles use a font-based encoding called Luit. For our experiments we transliterate the texts to a normalised Roman encoding using transliteration software developed by us. We manually tag a part of this corpus, $\operatorname{Tr}$, consisting of nearly 10,000 words for training. We use other portions of Corpus Asm for testing the tagger.

There was no tagset for Assamese before we started the project reported in this paper. Due to the morphological richness of the language, many words of Assamese occur in secondary forms in texts. This increases the number of POS tags that needed for the language. Also, often there are differences of opinion among linguists on the tags that may be associated with certain words in texts. We developed a tagset after in-depth consultation with linguists and manually tagged text segments of nearly 10,000 words according to their guidance. To make the tagging process easier we have subcategorised each category of noun and personal pronoun based on six case endings (viz, nominative, accussative, instumental, dative, genitive and locative) and two numbers.

We have used HMM

(Dermatas and Kokkinakis, 1995) and the Viterbi algorithm (1967) in developing our POS tagger. HMM/Viterbi approach is the most useful method, when pretagged corpus is not available. First, in the training phase, we have manually tagged the Tr part of the corpus using the tagset discussed above. Then, we build four database tables using probabilities extracted from the manually tagged corpus- word-probability table, previoustag-probability table, starting-tag-probability table and affix-probability table.

For testing, we consider three text segments, $A$, $B$ and $C$, each of about 1000 words. First the input text is segmented into sentences. Each sentence is parsed individually. Each word of a sentence is stored in an array. After that, each word is searched in the word-probability table. If the word is unknown, its possible affixes are extracted
Table 2: POS tagging results with small corpora. Size of training words : 10000, UWH : Unknown word handling, UPH : Unknown proper noun handling

\begin{tabular}{|c|c|c|c|c|}
\hline $\begin{array}{c}\text { Test } \\
\text { set }\end{array}$ & Size & $\begin{array}{c}\text { Average } \\
\text { accuracy }\end{array}$ & $\begin{array}{c}\text { UDH } \\
\text { accuracy }\end{array}$ & $\begin{array}{c}\text { UPH } \\
\text { accuracy }\end{array}$ \\
\hline A & 992 & $84.68 \%$ & $62.8 \%$ & $42.0 \%$ \\
\hline B & 1074 & $89.94 \%$ & $67.54 \%$ & $53.96 \%$ \\
\hline C & 1241 & $86.05 \%$ & $85.64 \%$ & $26.47 \%$ \\
\hline
\end{tabular}

Table 3: Comparison of our result with other HMM based model.

\begin{tabular}{|l|l|l|}
\hline Author & Language & $\begin{array}{l}\text { Average } \\
\text { accuracy }\end{array}$ \\
\hline Toutanova et al.(2003) & English & $97.24 \%$ \\
\hline Banko and Moore(2004) & English & $96.55 \%$ \\
\hline Dandapat and Sarkar(2006) & Bengali & $84.37 \%$ \\
\hline Rao et al.(2007) & Hindi & $76.34 \%$ \\
& Bengali & $72.17 \%$ \\
& Telegu & $53.17 \%$ \\
\hline \multirow{2}{*}{ Rao and Yarowsky(2007) } & Hindi & $70.67 \%$ \\
& Bengali & $65.47 \%$ \\
& Telegu & $65.85 \%$ \\
\hline Sastry et al.(2007) & Hindi & $69.98 \%$ \\
& Bengali & $67.52 \%$ \\
\hline \multirow{2}{*}{ Ekbal et al.(2007) } & Telegu & $68.32 \%$ \\
\hline Ours & Hindi & $71.65 \%$ \\
& Bengali & $80.63 \%$ \\
& Telegu & $53.15 \%$ \\
\hline
\end{tabular}

and searched in the affix-probability table. From this search, we obtain the probable tags and their corresponding probabilities for each word. All these probable tags and the corresponding probabilities are stored in a two dimensional array which we call the lattice of the sentence. If we do not get probable tags and probabilities for a certain word from these two tables we assign tag $\mathrm{CN}$ (Common Noun) and probability 1 to the word since occurrence of $\mathrm{CN}$ is highest in the manually tagged corpus. After forming the lattice, the Viterbi algorithm is applied to the lattice that yields the most probable tag sequence for that sentence. After that next sentence is taken and the same procedure is repeated.

\section{Experimental Evaluation}

The results using the three test segments are summarised in Table 2. The evaluation of the results require intensive manual verification effort. Larger training corpora is likely to produce more accurate results. More reliable results can be obtained using larger test corpora. Table 3 compares our result with other HMM based reported work. Form the table it is clear that 
Toutanova et al. (2003) obtained the best result for English (97.24\%). Among HMM based experiments reported on Indian languages, we have obtained the best result (86.89\%). This work is ongoing and the corpus size and the amount of tagged text are being increased on a regular basis.

The accuracy of a tagger depends on the size of tagset used, vocabulary used, and size, genre and quality of the corpus used. Our tagset containing 172 tags is rather big compared to other Indian language tagsets. A smaller tagset is likely to give more accurate result, but may give less information about word structure and ambiguity. The corpora for training and testing our tagger are taken form an Assamese daily newspaper Asomiya Pratidin, thus they are of the same genre.

\section{Conclusion \& Future work}

We have achieved good POS tagging results for Assamese, a fairly widely spoken language which had very little prior computational linguistic work. We have obtained an average tagging accuracy of $87 \%$ using a training corpus of just 10000 words. Our main achievement is the creation of the Assamese tagset that was not available before starting this project. We have implemented an existing method for POS tagging but our work is for a new language where an annotated corpora and a pre-defined tagset were not available.

We are currently working on developing a small and more compact tagset. We propose the following additional work for improved performance. First, the size of the manually tagged part of the corpus will have to be increased. Second, a suitable procedure for handling unknown proper nouns will have to be developed. Third, if this system can be expanded to trigrams or even n-grams using a larger training corpus, we believe that the tagging accuracy will increase.

\section{Acknowledgemnt}

We would like to thank Dr. Jyotiprakash Tamuli, Dr. Runima Chowdhary and Dr. Madhumita Barbora for their help, specially in making the Assamese tagset.

\section{References}

Avinesh PVS \& Karthik G. POS tagging and chunking using Conditional Random Field and Transformation based learning. IJCAI-07 workshop on Shallow Parsing for South Asian Languages. 2007.

Banko, M., \& Robert Moore, R. Part of speech tagging in context. 20th International Conference on Computational Linguistics. 2004.

Dandapat, S. Part-of-Speech Tagging and Chunking with Maximum Entropy Model. Workshop on Shallow Parsing for South Asian Languages. 2007.

Dandapat, S., \& Sarkar, S. Part-of-Speech Tagging for Bengali with Hidden Markov Model. NLPAI ML workshop on Part of speech tagging and Chunking for Indian language. 2006.

Dermatas, S., \& Kokkinakis, G. Automatic stochastic tagging of natural language text. Computational Linguistics 21 : 137-163. 1995.

Ekbal, A., Mandal, S., \& Bandyopadhyay, S. POS tagging using HMM and rule based chunking . Workshop on Shallow Parsing for South Asian Languages. 2007.

Goswami, G. C. Asamīyā Vyākaraṇ Pravesh, Second edition. Bina Library, Guwahati. 2003.

http://shiva.iiit.ac.in/SPSAL2007. IJCAI-07 workshop on Shallow Parsing for South Asian Languages. Hyderabad, India.

Pammi, S.C., \& Prahallad, K. POS tagging and chunking using Decision Forests. Workshop on Shallow Parsing for South Asian Languages. 2007.

Rao, D., \& Yarowsky, D.. Part of speech tagging and shallow parsing of Indian languages. IJCAI-07 workshop on Shallow Parsing for South Asian Languages. 2007.

Rao, P.T., \& Ram, S.R., Vijaykrishna, R. \& Sobha L. A text chunker and hybrid pos tagger for Indian languages. IJCAI-07 workshop on Shallow Parsing for South Asian Languages. 2007.

Saha, G.K., Saha, A.B., \& Debnath, S. Computer Assisted Bangla Words POS Tagging. Proc. International Symposium on Machine Translation NLP \& TSS. 2004.

Sastry, G.M.R., Chaudhuri, S., \& Reddy, P.N. A HMM based part-of-speech and statistical chunker for 3 Indian languages. IJCAI-07 workshop on Shallow Parsing for South Asian Languages. 2007.

Sharma, U., Kalita, J. \& Das, R. K. Acquisition of Morphology of an Indic language from text corpus. ACM TALIP 2008.

Singh, S., Gupta K., Shrivastava, M., \& Bhattacharyya, P. Morphological richness offsets resource demandexperiences in constructing a POS tagger for Hindi. COLING/ACL. 2006.

Toutanova, K., Klein, D., Manning, C.D. \& Singer, Y. Feature-Rich part-of-speech tagging with a Cyclic Dependency Network. HLT-NAACL. 2003.

Viterbi, A.J. Error bounds for convolutional codes and an asymptotically optimum decoding algorithm. IEEE Transaction on Information Theory 61(3) : 268-278. 1967. 